\title{
Conjugate Pairs of Subfactors and Entropy for Automorphisms
}

\author{
Marie Choda \\ November 1, 2018 \\ Osaka Kyoiku University, Asahigaoka, Kashiwara 582-8582, Japan \\ marie@cc.osaka-kyoiku.ac.jp
}

\begin{abstract}
Based on the fact that, for a subfactor $N$ of a $\mathrm{II}_{1}$ factor $M$, the first non-trivial Jones index is 2 and then $M$ is decomposed as the crossed product of $N$ by an outer action of $\mathbb{Z}_{2}$, we study pairs $\left\{N, u N u^{*}\right\}$ from a view point of entropy for two subalgebras of $M$ with a connection to the entropy for automorphisms, where the inclusion of $\mathrm{II}_{1}$ factors $N \subset M$ is given as $M$ is the crossed product of $N$ by a finite group of outer automorphisms and $u$ is a unitary in $M$.
\end{abstract}

keywords : Subfactor, entropy, conjugate.

Mathematics Subject Classification 2000: 46L55; 46L35

\section{Introduction}

For two von Neumann subalgebras $A$ and $B$ of a finite von Neumann algebra $M$, in the previous paper [4] we gave a modified constant $h(A \mid B)$ of the Connes-Størmer relative entropy $H(A \mid B)$ in [7] (cf. [18] ). The aim was to see the entropy for unistochastic matrices from the viewpoint of the operator algebras and we showed among others that $h\left(D \mid u D u^{*}\right)=H(b(u))$, where $D$ is the algebra of the diagonal matrices in the $n \times n$ complex matrices $M_{n}(\mathbb{C})$, and where $H(b(u))$ is the entropy in [21] for the unistochastic matrix $b(u)$ induced by a unitary $u$ in $M_{n}(\mathbb{C})$, and in general, it does not holds that $H\left(D \mid u D u^{*}\right)=H(b(u))$ (see, for example [17] ). 
In this paper, we replace the type $\mathrm{I}_{n}$ factor $M_{n}(\mathbb{C})$ to a $\mathrm{II}_{1}$ factor $M$. The above relation in [4] (cf. [15]) suggests us that if $A$ and $B$ are maximal abelian subalgebras of a $\mathrm{II}_{1}$ factor $M$, then $h(A \mid B)$ is not necessarily finite. In order to discuss on a subalgebra $A$ of $M$ with $h\left(A \mid u A u^{*}\right)<\infty$ for all unitaries $u$ in $M$, we pick up here a subfactor $N \subset M$ with the Jones index $[M: N]<\infty([11])($ cf. [8]).

Let $M$ be a type $\mathrm{II}_{1}$ factor, and let $N$ be a subfactor of $M$ such that $[M: N]=2$ which is the simplest, nontrivial unique subfactor of $M$ up to conjugacy. Then $M$ is decomposed into the crossed product of $N$ by an outer automorphism with the period 2. Based on this fact, we study the set of the values $h\left(N \mid u N u^{*}\right)$ for the inclusion of factor-subfactor $N \subset M$, with a connection to the inner automorphisms $A d u$, where $M$ is given as the crossed product $N \rtimes_{\alpha} G$ of a type $\mathrm{II}_{1}$ factor $N$ by a finite group $G$ with respect to an outer action $\alpha$ and $u$ is a unitary in $M$.

First, for two von Neumann subalgebras $A$ and $B$ of a finite von Neumann subalgebra $M$, we show, in Corollary 2.2 .3 below, that if $E_{A} E_{B}=E_{B} E_{A}$ (which is called the commuting square condition in the sense of [9]) then $H(A \mid B)=h(A \mid B)$, where $E_{A}$ is the conditional expectation of $M$ onto $A$.

We give an extended notion $H_{N}(A d u)$ of $H(b(u))$ to the inner automorphisms $A d u$ in Definition 3.1.1, and we show that $h\left(N \mid u N u^{*}\right) \leq H_{N}(A d u)$ in Theorem 3.1.4.

The inner conjugacy class of $N$ is rich from the view point of the values of $h\left(N \mid u N u^{*}\right)$, that is, in the special case of $G=\mathbb{Z}_{2}$, keeping the fact that $h\left(N \mid u N u^{*}\right) \leq H\left(M \mid u N u^{*}\right)=\log 2$ for all unitary $u \in M$ in mind, we have that $\left\{h\left(N \mid u N u^{*}\right): u \in M\right.$ a unitary $\}=[0, \log 2]$ in Theorem 3.2.3.

\section{Preliminaries}

In this section, we summarize, for future reference, notations, terminologies and basic facts.

Let $M$ be a finite von Neumann algebra, and let $\tau$ be a faithful normal tracial state. For each von Neumann subalgebra $A$, there is a unique $\tau$ preserving conditional expectation $E_{A}: M \rightarrow A$. 


\subsection{Connes-Størmer relative entropy}

Let $S$ be the set of all finite families $\left(x_{i}\right)_{i}$ of positive elements in $M$ with $1=\sum_{i} x_{i}$. Let $A$ and $B$ be two von Neumann subalgebras of $M$. The relative entropy $H(A \mid B)$ is defined by Connes and $\operatorname{Størmer}([7])$ as

$$
H(A \mid B)=\sup _{\left(x_{i}\right) \in S} \sum_{i}\left(\tau \eta E_{B}\left(x_{i}\right)-\tau \eta E_{A}\left(x_{i}\right)\right) .
$$

Here, $\eta$ is the function defined by

$$
\eta(t)=-t \log t,(0<t \leq 1) \quad \text { and } \eta(0)=0 .
$$

Let $\phi$ be a normal state on $M$. Let $\Phi$ be the set of all finite families $\left(\phi_{i}\right)_{i}$ of positive linear functionals on $M$ with $\phi=\sum_{i} \phi_{i}$. The relative entropy $H_{\phi}(A \mid B)$ of $A$ and $B$ with respect to $\phi$ is given by Connes ([6]) as

$$
H_{\phi}(A \mid B)=\sup _{\left(\phi_{i}\right) \in \Phi} \sum_{i}\left(S\left(\left.\phi_{i}\right|_{A},\left.\phi\right|_{A}\right)-S\left(\left.\phi_{i}\right|_{B},\left.\phi\right|_{B}\right)\right)
$$

and if $\phi=\tau$ then $H_{\tau}(A \mid B)=H(A \mid B)$. Here $S(\phi, \psi)$ is the relative entropy for positive linear functionals $\phi$ and $\psi$ on $M$ (cf. [14, 16]).

\subsection{Modified relative entropy for two subalgebras.}

We modified in [4] the Connes-Størmer relative entropy for a pair of subalgebras as follows :

Let $A$ and $B$ be two von Neumann subalgebras of $M$. Let $S$ be the set of all finite families $\left(x_{i}\right)$ of positive elements in $M$ with $1=\sum_{i} x_{i}$. The conditional relative entropy $h(A \mid B)$ of $A$ and $B$ corresponding $H(A \mid B)$ is given as

$$
h(A \mid B)=\sup _{\left(x_{i}\right) \in S} \sum_{i}\left(\tau \eta E_{B}\left(E_{A}\left(x_{i}\right)\right)-\tau \eta E_{A}\left(x_{i}\right)\right) .
$$

Let $S(A) \subset S$ be the set of all finite families $\left(x_{i}\right)$ of positive elements in $A$ with $1=\sum_{i} x_{i}$. Then it is clear that

$$
h(A \mid B)=\sup _{\left(x_{i}\right) \in S(A)} \sum_{i}\left(\tau \eta E_{B}\left(x_{i}\right)-\tau \eta\left(x_{i}\right)\right) .
$$


Let $S^{\prime}(A) \subset S(A)$ be the set of all finite families $\left(x_{i}^{\prime}\right)$ with each $x_{i}^{\prime}$ a scalar multiple of a projection in $A$. Then

$$
h(A \mid B)=\sup _{\left(x_{i}^{\prime}\right) \in S^{\prime}(A)} \sum_{i}\left(\tau \eta E_{B}\left(x_{i}^{\prime}\right)-\tau \eta\left(x_{i}^{\prime}\right)\right) .
$$

The conditional relative entropy of $A$ and $B$ with respect to $\phi$ corresponding $H_{\phi}(A \mid B)$ is given as

$$
h_{\phi}(A \mid B)=\sup _{\left(\phi_{i}\right) \in \Phi} \sum_{i}\left(S\left(\left.\phi_{i}\right|_{A},\left.\phi\right|_{A}\right)-S\left(\left.\left(\phi_{i} \circ E_{A}\right)\right|_{B},\left.\left(\phi \circ E_{A}\right)\right|_{B}\right) .\right.
$$

If we let $\Phi(A) \subset \Phi$ be the set of all finite families $\left(\phi_{i}^{\prime}\right)$ in $\Phi$ with each $\phi_{i}^{\prime}=\phi_{i}^{\prime} \circ E_{A}$, then

$$
h_{\phi}(A \mid B)=\sup _{\left(\phi_{i}^{\prime}\right) \in \Phi(A)} \sum_{i}\left(S\left(\left.\phi_{i}^{\prime}\right|_{A},\left.\phi\right|_{A}\right)-S\left(\left.\phi_{i}^{\prime}\right|_{B},\left.\left(\phi \circ E_{A}\right)\right|_{B}\right) .\right.
$$

We give conditions for that $h_{\phi}(A \mid B)=H_{\phi}(A \mid B)$ in Corollary 2.2.4 below, and show relations for $h_{\phi}(A \mid B), H_{\phi}(A \mid B), H_{\phi}(A)$ and $h_{\phi}(A)$ in Theorem 2.2.2, where $h_{\phi}(A)$ is given by modifing $H_{\phi}(A)$ in [6] for a von Neumann subalgebra $A$ of $M$ (cf. [14]), that is

$$
h_{\phi}(A)=\sup _{\left(\phi_{i}\right) \in \Phi(A)} \sum_{i}\left(\eta\left(\phi_{i}(1)\right)+S\left(\left.\phi_{i}\right|_{A},\left.\phi\right|_{A}\right)\right)
$$

and

$$
H_{\phi}(A)=\sup _{\left(\phi_{i}\right) \in \Phi} \sum_{i}\left(\eta\left(\phi_{i}(1)\right)+S\left(\left.\phi_{i}\right|_{A},\left.\phi\right|_{A}\right)\right) .
$$

Cleary, we have that $0 \leq h_{\phi}(A) \leq H_{\phi}(A)$. In the case of $\phi$ is the trace $\tau$, $h_{\phi}(A)=h(A)$, which is given as

$$
h(A)=\sup _{\left(x_{i}\right) \in S(A)} \sum_{i}\left(\eta\left(\tau\left(x_{i}\right)\right)-\tau \eta\left(x_{i}\right)\right) .
$$

We need the following lemma in order to prove Theorem 2.2.2, in which we show relations among $H_{\phi}(A), h_{\phi}(A), H_{\phi}(A \mid B)$ and $H_{\phi}(A \mid B)$ :

Lemma 2.2.1. Let $A$ and $B$ be von Neumann subalgebras of a finite von Neumann algebra $M$, and let $\psi, \phi$ be positive linear functionals on $M$. If $E_{A} E_{B}=E_{B} E_{A}$, then

$$
S\left(\left.\left(\psi \circ E_{A}\right)\right|_{B},\left.\left(\phi \circ E_{A}\right)\right|_{B}\right)=S\left(\left.\left(\psi \circ E_{A}\right)\right|_{A \cap B},\left.\left(\phi \circ E_{A}\right)\right|_{A \cap B}\right) .
$$


Proof. Relative entropy for positive linear functionals $\psi, \phi$ on a unital $C^{*}$ algebra $C$ is expressed in [13] by

$$
S(\psi, \phi)=\sup _{n \in \mathbb{N}} \sup _{x}\left\{\psi(1) \log n-\int_{1 / n}^{\infty}\left(\psi\left(y(t)^{*} y(t)\right)+\frac{1}{t} \phi\left(x(t) x(t)^{*}\right)\right) \frac{d t}{t}\right\}
$$

where $x(t):\left(\frac{1}{n}, \infty\right) \rightarrow C$ is a step function with finite range, and $y(t)=$ $1-x(t)$.

Let $x(t):\left(\frac{1}{n}, \infty\right) \rightarrow B$ be a step function with finite range, then $E_{A}(x(t))$ : $\left(\frac{1}{n}, \infty\right) \rightarrow A \cap B$ is a step function with finite range because of that $E_{A} E_{B}=$ $E_{B} E_{A}$. Since $E_{A}(x)^{*} E_{A}(x) \leq E_{A}\left(x^{*} x\right)$ for all $x \in M$, we have that

$$
\begin{aligned}
\psi(1) & \log n-\int_{1 / n}^{\infty}\left(\psi \circ E_{A}\left(y(t)^{*} y(t)\right)+\frac{1}{t} \phi \circ E_{A}\left(x(t) x(t)^{*}\right)\right) \frac{d t}{t} \\
& \leq \psi(1) \log n-\int_{1 / n}^{\infty}\left(\psi\left(E_{A}(y(t))^{*} E_{A}(y(t))\right)+\frac{1}{t} \phi\left(E_{A}(x(t)) E_{A}\left(x(t)^{*}\right)\right)\right) \frac{d t}{t} \\
& \leq S\left(\left.\left(\psi \circ E_{A}\right)\right|_{A \cap B},\left.\left(\phi \circ E_{A}\right)\right|_{A \cap B}\right) .
\end{aligned}
$$

This implies that

$$
S\left(\left.\left(\psi \circ E_{A}\right)\right|_{B},\left.\left(\phi \circ E_{A}\right)\right|_{B}\right) \leq S\left(\left.\left(\psi \circ E_{A}\right)\right|_{A \cap B},\left.\left(\phi \circ E_{A}\right)\right|_{A \cap B}\right) .
$$

Since the opposite inequality is clear, we have the equality.

Theorem 2.2.2. Let $M$ be a finite von Neumann algebra with a normal faithful tracial state $\tau$. Let $\phi$ be a normal state of $M$, and let $A, B$ be von Neumann subalgebras of $M$. Then

(1) $h_{\phi}(A \mid B) \leq h_{\phi}(A \mid \mathbb{C} \mathbf{1})=h_{\phi}(A)$.

(2) Assume that $E_{A} E_{B}=E_{B} E_{A}$. Then $h_{\phi}(A \mid B)=h_{\phi}(A \mid A \cap B)$.

Hence, if $A \cap B=\mathbb{C}$, then $h_{\phi}(A \mid B)=h_{\phi}(A)$.

(3) If $E_{A} E_{B}=E_{B} E_{A}$ and if $\phi=\phi \circ E_{A}$, then $H_{\phi}(A \mid B)=H_{\phi}(A \mid A \cap B)$.

Especially, if $A \cap B=\mathbb{C}$, then $H_{\phi}(A \mid B)=H_{\phi}(A)$.

(4) If $B \subset A$, then

$$
H_{\phi}(A \mid B)=h_{\phi}(A \mid B) .
$$

Especially, $H_{\phi}(A)=h_{\phi}(A)$. 
Proof. (1) Let $\phi, \psi$ be normal states of $M$, and let $\left(\phi_{i}\right)_{i} \in \Phi$. Then $\left.\left(\phi \circ E_{A}\right)\right|_{B}$ and $\left.\frac{1}{\phi_{i}(1)}\left(\phi_{i} \circ E_{A}\right)\right|_{B}$ are states of $B$ so that

$$
S\left(\left.\frac{1}{\phi_{i}(1)}\left(\phi_{i} \circ E_{A}\right)\right|_{B},\left.\left(\phi \circ E_{A}\right)\right|_{B}\right) \geq 0
$$

and

$$
\begin{aligned}
& S\left(\left.\frac{1}{\phi_{i}(1)}\left(\phi_{i} \circ E_{A}\right)\right|_{B},\left.\left(\phi \circ E_{A}\right)\right|_{B}\right) \\
& \quad=\frac{1}{\phi_{i}(1)} S\left(\left.\phi_{i} \circ E_{A}\right|_{B},\left.\left(\phi \circ E_{A}\right)\right|_{B}\right)-\phi_{i}(1) \eta\left(\frac{1}{\phi_{i}(1)}\right) \\
& \quad=\frac{1}{\phi_{i}(1)} S\left(\left.\phi_{i} \circ E_{A}\right|_{B},\left.\left(\phi \circ E_{A}\right)\right|_{B}\right)-\log \left(\phi_{i}(1)\right) .
\end{aligned}
$$

Hence

$$
-S\left(\left.\left(\phi_{i} \circ E_{A}\right)\right|_{B},\left.\left(\phi \circ E_{A}\right)\right|_{B}\right) \leq \eta\left(\phi_{i}(1)\right),
$$

and if $B=\mathbb{C} 1$ then the equality holds because $\left.\frac{1}{\phi_{i}(1)} \phi_{i}\right|_{\mathbb{C} \mathbf{1}}=\left.\phi\right|_{\mathbb{C} \mathbf{1}}$. These imply that

$$
\begin{aligned}
& h_{\phi}(A \mid B) \\
& \quad=\sup _{\left(\phi_{i}\right) \in \Phi} \sum_{i}\left(S\left(\left.\phi_{i}\right|_{A},\left.\phi\right|_{A}\right)-S\left(\left.\left(\phi_{i} \circ E_{A}\right)\right|_{B},\left.\left(\phi \circ E_{A}\right)\right|_{B}\right)\right) \\
& \quad \leq \sup _{\left(\phi_{i}\right) \in \Phi} \sum_{i}\left(S\left(\left.\phi_{i}\right|_{A},\left.\phi\right|_{A}\right)+\eta\left(\phi_{i}(1)\right)\right) \\
& \quad=h_{\phi}(A),
\end{aligned}
$$

and the equality holds if $B=\mathbb{C} 1$.

(2) Assume that $E_{A} E_{B}=E_{B} E_{A}$, then by lemma 2.2.1, we have that

$$
\begin{aligned}
& h_{\phi}(A \mid B) \\
& \quad=\sup _{\left(\phi_{i}\right) \in \Phi} \sum_{i}\left(S\left(\left.\phi_{i}\right|_{A},\left.\phi\right|_{A}\right)-S\left(\left.\left(\phi_{i} \circ E_{A}\right)\right|_{B},\left.\left(\phi \circ E_{A}\right)\right|_{B}\right)\right) \\
& \quad=\sup _{\left(\phi_{i}\right) \in \Phi} \sum_{i}\left(S\left(\left.\phi_{i}\right|_{A},\left.\phi\right|_{A}\right)-S\left(\left.\left(\phi_{i} \circ E_{A}\right)\right|_{A \cap B},\left.\left(\phi \circ E_{A}\right)\right|_{A \cap B}\right)\right) \\
& \quad=h_{\phi}(A \mid A \cap B) .
\end{aligned}
$$

Since $h_{\phi}(A \mid B)$ is decreasing in $B$, it implies that

$$
h_{\phi}(A \mid B)=h_{\phi}(A \mid A \cap B) .
$$


Hence, if $A \cap B=\mathbb{C} 1$, then $h_{\phi}(A \mid B)=h_{\phi}(A \mid \mathbb{C} 1)=h_{\phi}(A)$.

(3) Assume that $E_{A} E_{B}=E_{B} E_{A}$ and that $\phi \circ E_{A}=\phi$. Let $\left(\phi_{i}\right)_{i} \in \Phi$, then $\left(\phi_{i} \circ E_{A}\right)_{i} \in \Phi$ and we have that

$$
\begin{aligned}
H_{\phi}(A \mid B) & \\
\quad & \sup _{\left(\phi_{i}\right) \in \Phi} \sum_{i}\left(S\left(\left.\phi_{i}\right|_{A},\left.\phi\right|_{A}\right)-S\left(\left.\phi_{i}\right|_{B},\left.\phi\right|_{B}\right)\right) \\
& \geq \sup _{\left(\phi_{i}\right) \in \Phi} \sum_{i}\left(S\left(\left.\left(\phi_{i} \circ E_{A}\right)\right|_{A},\left.\phi\right|_{A}\right)-S\left(\left.\left(\phi_{i} \circ E_{A}\right)\right|_{B},\left.\phi\right|_{B}\right)\right) \\
& =\sup _{\left(\phi_{i}\right) \in \Phi} \sum_{i}\left(S\left(\left.\phi_{i}\right|_{A},\left.\phi\right|_{A}\right)-S\left(\left.\left(\phi_{i} \circ E_{A}\right)\right|_{B},\left.\left(\phi \circ E_{A}\right)\right|_{B}\right)\right) \\
& =\sup _{\left(\phi_{i}\right) \in \Phi} \sum_{i}\left(S\left(\left.\phi_{i}\right|_{A},\left.\phi\right|_{A}\right)-S\left(\left.\left(\phi_{i} \circ E_{A}\right)\right|_{A \cap B},\left.\left(\phi \circ E_{A}\right)\right|_{A \cap B}\right)\right) \\
& =\sup _{\left(\phi_{i}\right) \in \Phi} \sum_{i}\left(S\left(\left.\phi_{i}\right|_{A},\left.\phi\right|_{A}\right)-S\left(\left.\phi_{i}\right|_{A \cap B},\left.\phi\right|_{A \cap B}\right)\right) \\
= & H_{\phi}(A \mid A \cap B) .
\end{aligned}
$$

In general, $H_{\phi}(A \mid A \cap B) \geq H_{\phi}(A \mid B)$ so that

$$
H_{\phi}(A \mid B)=H_{\phi}(A \mid A \cap B) .
$$

Especially, $H(A \mid B)=H(A \mid A \cap B)$ (cf. [22]), and if $A \cap B=\mathbb{C}$, then $H_{\phi}(A \mid A \cap B)=H_{\phi}(A \mid \mathbb{C} 1)=H_{\phi}(A)$ so that

$$
H_{\phi}(A \mid B)=H_{\phi}(A) .
$$

(4) If $B \subset A$, then

$$
\begin{aligned}
H_{\phi}(A \mid B) & \\
\quad= & \sup _{\left(\phi_{i}\right) \in \Phi} \sum_{i}\left(S\left(\left.\phi_{i}\right|_{A},\left.\phi\right|_{A}\right)-S\left(\left.\phi_{i}\right|_{B},\left.\phi\right|_{B}\right)\right) \\
& =\sup _{\left(\phi_{i}\right) \in \Phi} \sum_{i}\left(S\left(\left.\phi_{i}\right|_{A}, \phi||_{A}\right)-S\left(\left.\left(\phi_{i} \circ E_{A}\right)\right|_{B},\left.\left(\phi \circ E_{A}\right)\right|_{B}\right)\right) \\
& =h_{\phi}(A \mid B) .
\end{aligned}
$$

By combining with $(1)$, we have $H_{\phi}(A)=h_{\phi}(A)$. 
Corollary 2.2.3. Assume that $E_{A} E_{B}=E_{B} E_{A}$. Then $H(A \mid B)=h(A \mid B)$. Moreover, if $\phi=\phi \circ E_{A}$, then

$$
H_{\phi}(A \mid B)=h_{\phi}(A \mid B) .
$$

Proof. Assume that $E_{A} E_{B}=E_{B} E_{A}$ and that $\phi=\phi \circ E_{A}$. Then by using Theorem 2.2.2 (3), (4) and (2), we have that

$$
H_{\phi}(A \mid B)=H_{\phi}(A \mid A \cap B)=h_{\phi}(A \mid A \cap B)=h_{\phi}(A \mid B) .
$$

Since $E_{A}$ is the $\tau$-conditional expectation, $\tau=\tau \circ E_{A}$, hence

$$
H(A \mid B)=h(A \mid B) .
$$

\section{$3 \quad$ Inner conjugate subfactors}

Connes-Størmer defined the entropy $H(\alpha)$ for a trace preserving automorphism $\alpha$ of a finite von Neumann algebra in [7]. The definition is arivable for a trace preserving $*_{\text {-endomorphism too. }}$

For a trace preserving *-endomorphism $\sigma$ of a finite von Neumann algebra $N$, it was shown a relation between the entropy $H(\sigma)$ for $\sigma$ and the relative entropy $H(N \mid \sigma(N))$ in the papers [1, 2, 3, 10, 20] (cf. [14]). The relation is, roughly speaking, that

$$
H(\sigma)=\frac{1}{2} H(N \mid \sigma(N))
$$

under a certain condition. Such a *-endomorphism $\sigma$ can be extended offten to an automorphism $\alpha$ of a finite von Neumann algebra $M$ which contains $N$ as a von Neumann subalgebra. Some examples of such endomorphisms appeared in a relation to Jones index theory of subfactors. In [2], we studied a nice class of such a *-endomorphism $\sigma$ of a type $\mathrm{II}_{1}$ factor $N$ which is extendable to an automorphism $\alpha$ of the big type $\mathrm{II}_{1}$ factor $M$ obtained by the basic construction from $N \supset \sigma(N)$. We called such a $\sigma$ basic ${ }^{*}$-endomorphism and 
showed that $H(\alpha)=\frac{1}{2} H(N \mid \sigma(N))$. Since $\sigma(N) \subset N$, we have by Theorem 2.2 .2 (4) that $H(N \mid \sigma(N))=h(N \mid \sigma(N))$ so that

$$
H(\alpha)=\frac{1}{2} H(N \mid \sigma(N))=\frac{1}{2} h(N \mid \sigma(N))=\frac{1}{2} h(N \mid \alpha(N)) .
$$

This means that for an automorphism $\alpha$ of a $\mathrm{II}_{1}$ factor $M$ we may be able to choose a subfactor $N \subset M$ such that the entropy for $\alpha$ is given from $h(N \mid \alpha(N))$.

Our study in this section is motivated by these results. The above automorphism $\alpha$ arising from a ${ }^{*}$-endomorphism as is outer. Here, we discuss by replacing the $\alpha$ to inner automorphisms $A d u$ and the entropy $H(\alpha)$ to the entropy $H_{N}(A d u)$ defined below.

\subsection{Entropy for Inner Automorphisms with respect to Subfactors}

Let $N$ be a type $\mathrm{II}_{1}$ factor with the canonical trace $\tau$ and let $G$ be a finite group. Let $\alpha$ be an outer action of $G$ on $N$, so that for all $g \in G, g \neq 1$ if $\alpha_{g}(x) a=a x$ for all $x \in N$, then $a=0$. Hereafter, we let $M$ be the crossed product of $N$ by $G$ with respect to $\alpha$ :

$$
M=N \rtimes_{\alpha} G
$$

We identify $N$ with the von Neumann subalgebra embedded in $M$, and denote by $v$ the unitary representation of $G$ in $M$ such that every $v_{g}$ is a unitary in $M$ with

$$
\alpha_{g}(x)=v_{g} x v_{g}^{*}, \quad(x \in N, g \in G) .
$$

Then every $x \in M$ is written by the Fourier expansion

$$
x=\sum_{g \in G} x_{g} v_{g}, \quad\left(x_{g} \in N\right)
$$

and $x_{g}=E_{N}\left(x v_{g}^{*}\right)$. A $u \in M$ is a unitary if and only if

$$
\sum_{g \in G} u_{h g} \alpha_{h}\left(u_{g}^{*}\right)=\delta_{h, 1} \quad \text { and } \quad \sum_{g \in G} \alpha_{g}^{-1}\left(u_{g}^{*} u_{g h}\right)=\delta_{h, 1}
$$

where we denote the identity of $G$ by 1 . This imply that $\sum_{g \in G} \tau\left(u_{g} u_{g}^{*}\right)=1$, and we can put as the followings : 
Definition 3.1.1. The entropy of the inner automorphism Adu of $M$ with respect to $N$ is given by

$$
H_{N}(A d u)=\sum_{g \in G} \eta \tau\left(u_{g} u_{g}^{*}\right)
$$

Comment 3.1.2. Each $x \in M$ is representated as the matrix $x=(x(g, h))_{g h}$ indexed by the elements of $G$. Here $x(g, h) \in N$ for all $g, h$ in $G$, and $x(g, h)=$ $\alpha_{g}^{-1}\left(E_{N}\left(x v_{h}^{*}\right)\right)=\alpha_{g}^{-1}\left(x_{h}\right)$. The entropy $H(b(u))$ defined in [21] is written as

$$
H(b(u))=\frac{1}{n} \sum_{i, j} \eta\left(|u(i, j)|^{2}\right),
$$

when $b(u)$ is the unistochastic matrix induced by a unitary $u=(b(i, j))_{i j}$ in $M_{n}(\mathbb{C})$. A matrix representation for an $x$ in $M_{n}(\mathbb{C})$ is depend on the diagonal matrix algebra. In that sense, we consider the notion of $H_{N}(A d u)$ corresponds to the notion of the entropy for a unistochastic matrix.

Lemma 3.1.3. (1) If Adu and Adw are conjugate, then $H_{N}(A d u)=H_{N}(A d w)$.

(2) If $\theta=$ Adu for some unitary $u \in M$, then $H_{N}\left(\theta^{-1}\right)=H_{N}(\theta)$.

Proof. (1) Assume $A d u=\theta A d w \theta^{-1}$ for some automorphism $\theta$ of $M$. Then $\theta(w)=\lambda u$ for some complex number $\lambda$ with $|\lambda|=1$ and so $\eta \tau\left(w_{g} w_{g}^{*}\right)=$ $\eta \tau\left(u_{g} u_{g}^{*}\right)$ which implies that $H_{N}(A d u)=H_{N}(A d w)$.

(2) Let $w \in M$ be a unitary with $A d w=\theta^{-1}$, then $w=\gamma u^{*}$ for some $\gamma \in \mathbb{T}$. For the expression that $w=\sum_{g} w_{g} v_{g}$, we have that $w_{g}=\gamma \alpha_{g}\left(u_{g^{-1}}^{*}\right)$ for all $g \in G$ so that

$$
H_{N}\left(\theta^{-1}\right)=\sum_{g} \eta \tau\left(w_{g} w_{g}^{*}\right)=\sum_{g} \eta \tau\left(u_{g} u_{g}^{*}\right)=H_{N}(\theta) .
$$

The $h\left(N \mid u N u^{*}\right)$ is bounded by $H_{N}(A d u)$ as follows :

Theorem 3.1.4. Assume that $N$ is a type $I I_{1}$ factor, $G$ is a finite group and $M=N \rtimes_{\alpha} G$ with respect to the outer action $\alpha$. Then for each unitary $u \in M$, we have that

$$
h\left(N \mid u N u^{*}\right) \leq H_{N}(A d u)=\sum_{g \in G} \eta \tau\left(u_{g} u_{g}^{*}\right) \leq \log |G|,
$$


where $|G|$ is the cardinarity of $G$.

Proof. Let $\left(\lambda_{i} p_{i}\right)_{i \in I} \in S^{\prime}(N)$ be a finite partition of the unity, that is,

$$
\sum_{i \in I} \lambda_{i} p_{i}=1
$$

where $\left(\lambda_{i}\right)_{i \in I}$ are positive numbers and $\left(p_{i}\right)_{i \in I}$ are projections in $N$. For a given $\varepsilon$, choose an $\epsilon>0$ with with $2|G| \eta(\epsilon)<\min \{\varepsilon, 1 / e\}$. There exist mutually orthogonal projections $\left(q_{i, k}\right)_{k} \subset N$ and nonnegative numbers $\left(\alpha_{i, k}^{g}\right)_{k}$ which satisfy that

$$
p_{i}=\sum_{k} q_{i, k} \quad \text { and } \quad 0 \leq q_{i, k} u_{g} u_{g}^{*} q_{i, k}-\alpha_{i, k}^{g} q_{i, k} \leq \epsilon q_{i, k} .
$$

This is possible by the induction method of the spectral decompositions for $\left(p_{i} u_{g} u_{g}^{*} p_{i}\right)_{i \in I, g \in G}$, (see for example. [18, Proof of 4.3 Lemma]). In fact, letting $G=\left\{g_{1}, \cdots, g_{m}\right\}$ and by the spectral decomposition for $p_{i} u_{g_{1}} u_{g_{1}}^{*} p_{i} \in p_{i} N p_{i}$, we have mutually orthogonal projections $\left(q_{i, k}^{1}\right)_{k_{1}} \subset p_{i} N p_{i}$ and nonnegative numbers $\left(\alpha_{i, k_{1}}^{1}\right)_{k_{1}}$

$$
p_{i}=\sum_{k_{1}} q_{i, k_{1}}^{1} \quad \text { and } \quad 0 \leq q_{i, k_{1}}^{1} u_{g_{1}} u_{g_{1}}^{*} q_{i, k_{1}}^{1}-\alpha_{i, k_{1}}^{1} q_{i, k_{1}}^{1} \leq \epsilon q_{i, k_{1}}^{1}
$$

Next by the consideration for $q_{i, k_{1}}^{1} u_{g_{2}} u_{g_{2}}^{*} q_{i, k_{1}}^{1}$, we have a partition $\left(q_{i, k_{1}, k_{2}}^{2}\right)_{k_{2}}$ of $q_{i, k_{1}}^{1}$ and $\left(\alpha_{i, k_{1}, k_{2}}^{2}\right)_{k_{2}}$. Put $\alpha_{i, k}^{g_{j}}=\alpha_{i, k_{1}, \cdots, k_{j}}^{j}$ and $q_{i, k}=q_{i, k_{1}, \cdots, k_{m}}^{m}$. Then these satisfy the desired conditions.

Since $\eta(x+y) \leq \eta(x)+\eta(y),(x, y \in N), \eta$ is increasing on $[0,1 / e]$, and the family $\left(q_{i, k}\right)_{k}$ is mutually orthogonal, we have for $\epsilon$ with $\epsilon \leq 1 / e$

$$
\begin{aligned}
& \tau \eta\left(\sum_{k} q_{i, k} u_{g} u_{g}^{*} q_{i, k}\right) \\
& \quad \leq \tau \eta\left(\sum_{k}\left(q_{i, k} u_{g} u_{g}^{*} q_{i, k}-\alpha_{i, k}^{g} q_{i, k}\right)\right)+\tau \eta\left(\sum_{k} \alpha_{i, k}^{g} q_{i, k}\right) \\
& \quad \leq \eta(\epsilon) \tau\left(p_{i}\right)+\sum_{k} \eta\left(\alpha_{i, k}^{g}\right) \tau\left(q_{i, k}\right) .
\end{aligned}
$$

Hence, by the condition that $\sum_{i, k} \lambda_{i} \tau\left(q_{i, k}\right)=1$, the operator concavity of $\eta$ 
implies that

$$
\begin{aligned}
\sum_{i, g} & \lambda_{i} \tau \eta\left(\sum_{k} q_{i, k} u_{g} u_{g}^{*} q_{i, k}\right) \\
& \leq \sum_{i, k, g} \lambda_{i} \eta\left(\alpha_{i, k}^{g}\right) \tau\left(q_{i, k}\right)+|G| \eta(\epsilon) \\
& =\sum_{i, k} \lambda_{i} \tau\left(q_{i, k}\right) \sum_{g} \eta\left(\alpha_{i, k}^{g}\right)+|G| \eta(\epsilon) \\
& \leq \sum_{g} \eta\left(\sum_{i, k} \lambda_{i} \tau\left(q_{i, k}\right) \alpha_{i, k}^{g}\right)+|G| \eta(\epsilon) .
\end{aligned}
$$

Remark that for all $g \in G$

$$
\tau\left(u_{g} u_{g}^{*}\right)-\sum_{i, k} \lambda_{i} \alpha_{i, k}^{g} \tau\left(q_{i, k}\right)=\sum_{i, k} \lambda_{i} \tau\left(q_{i, k} u_{g} u_{g}^{*} q_{i, k}-\alpha_{i, k}^{g} q_{i, k}\right)
$$

and that

$$
0 \leq \sum_{i, k} \lambda_{i} \tau\left(q_{i, k} u_{g} u_{g}^{*} q_{i, k}-\alpha_{i, k}^{g} q_{i, k}\right) \leq \epsilon
$$

Then we have that

$$
0 \leq \tau\left(u_{g} u_{g}^{*}\right)-\sum_{i, k} \lambda_{i} \tau\left(q_{i, k}\right) \alpha_{i, k}^{g} \leq \epsilon, \quad(g \in G)
$$

so that

$$
\sum_{g} \eta\left(\sum_{i, k} \lambda_{i} \tau\left(q_{i, k}\right) \alpha_{i, k}^{g}\right) \leq \sum_{g} \eta \tau\left(u_{g} u_{g}^{*}\right)+|G| \eta(\epsilon) .
$$

Here we used the following inequality in [14, (2.8)]

$$
|\eta(s)-\eta(t)| \leq \eta(s-t) \text { for } 0 \leq s-t \leq \frac{1}{2} .
$$

Remark that $\sum_{g} u_{g} u_{g}^{*}=1$ and that for all $i$ the projections $\left(q_{i, k}\right)_{k}$ is mutually orthogonal. Hence by using the following fact that

$$
\tau \eta\left(u_{g}^{*} q_{i, k} u_{g}\right)=\tau \eta\left(q_{i, k} u_{g} u_{g}^{*} q_{i, k}\right),
$$


we have that

$$
\begin{aligned}
\sum_{i, k} & \left(\tau \eta E_{N}\left(u^{*} \lambda_{i} q_{i, k} u\right)-\tau \eta\left(\lambda_{i} q_{i, k}\right)\right) \\
& =\sum_{i, k}\left(\tau \eta\left(\sum_{g \in G} \alpha_{g}\left(u_{g}^{*} \lambda_{i} q_{i, k} u_{g}\right)-\eta\left(\lambda_{i}\right) \tau\left(q_{i, k}\right)\right)\right) \\
& \leq \sum_{i, k} \sum_{g}\left(\tau \eta\left(u_{g}^{*} \lambda_{i} q_{i, k} u_{g}\right)-\eta\left(\lambda_{i}\right) \tau\left(q_{i, k}\right)\right) \\
& =\sum_{i, k, g}\left(\eta\left(\lambda_{i}\right) \tau\left(u_{g}^{*} q_{i, k} u_{g}\right)+\lambda_{i} \tau \eta\left(u_{g}^{*} q_{i, k} u_{g}\right)-\sum_{i, k} \eta\left(\lambda_{i}\right) \tau\left(q_{i, k}\right)\right. \\
& =\sum_{i}\left(\eta\left(\lambda_{i}\right) \tau\left(p_{i} \sum_{g} u_{g} u_{g}^{*}\right)+\sum_{i, k, g} \lambda_{i} \tau \eta\left(u_{g}^{*} q_{i, k} u_{g}\right)-\sum_{i} \eta\left(\lambda_{i}\right) \tau\left(p_{i}\right)\right. \\
& =\sum_{i, k, g} \lambda_{i} \tau \eta\left(q_{i, k} u_{g} u_{g}^{*} q_{i, k}\right) \\
& =\sum_{i, g} \lambda_{i} \tau \eta\left(\sum_{k} q_{i, k} u_{g} u_{g}^{*} q_{i, k}\right) \\
& \leq \sum_{g} \eta \tau\left(u_{g} u_{g}^{*}\right)+2|G| \eta(\epsilon) .
\end{aligned}
$$

Thus

$$
\begin{aligned}
& h\left(N \mid u N u^{*}\right)=\sup _{\left(\lambda_{i} p_{i}\right) \in S^{\prime}(N)} \sum_{i}\left(\tau \eta E_{N}\left(u^{*} \lambda_{i} p_{i} u\right)-\tau \eta\left(\lambda_{i} p_{i}\right)\right) \\
& =\sup _{\left(\lambda_{i} q_{i k}\right)_{i, k}} \sum_{i, k}\left(\tau \eta E_{N}\left(u^{*} \lambda_{i} q_{i, k} u\right)-\tau \eta\left(\lambda_{i} q_{i, k}\right)\right) \\
& \quad \leq \sum_{g} \eta \tau\left(u_{g} u_{g}^{*}\right) .
\end{aligned}
$$

Since $\eta$ is a concave function, Theorem 3.1.4 implies the following :

Corollary 3.1.5. Assume that $N, G, u$ be as in Theorem 3.1.4 and that $h\left(N \mid u N u^{*}\right)=\log |G|$. Then $\tau\left(u_{g} u_{g}^{*}\right)=\frac{1}{|G|}$ for all $g \in G$.

Remark and Example 3.1.6. Let $A$ and $B$ be subalgebras of a type $\mathrm{II}_{1}$ factor $M$. Then $h(A \mid B) \leq H(A \mid B) \leq H(M \mid B)$, and if $B$ is a subfactor with 
$B^{\prime} \cap M=\mathbb{C}$ then $H(M \mid B)=\log [M: B]$ by [18] so that $h(A \mid B) \leq \log [M: B]$.

Størmer says that relative entropy can be viewed as a measure of distance between two subalgebras, which in the noncommutative case also measures their sizes and relative position.

Here, we give an example, which shows that $h(A \mid B)$ measures relative position and that some small size subalgebra $A_{G}$ can take the maximal value of $h(A \mid B)$, although the entropy $h(A \mid B)$ is increasing in $A$.

Assume that the finite group $G$ in Theorem 3.1.4 is abelian. Let $B=$ $u N u^{*}$. By taking the inner automorphism $A d u^{*}$, we may consider $M$ as the crossed product of $B$ by $G$ so that $x \in M$ has a unique expansion $x=$ $\sum_{g \in G} x_{g} v_{g},\left(x_{g} \in B\right)$. Let $A_{G}$ be the von Neumann algebra generated by the unitary group $v_{G}$, (that is, $|G|$ dimensional abelian algebra). Then

$$
h\left(A_{G} \mid B\right)=\log |G|=H(M \mid B)=\log [M: B] .
$$

In fact, it is clear that $h(A \mid B) \leq H(M \mid B)=\log [M: B]=\log |G|$. To show the opposite iniquality, let $\hat{G}$ be the character group of $G$. Given $\chi \in \hat{G}$, let

$$
p_{\chi}=\frac{1}{|G|} \sum_{g \in G} \chi_{g} v_{g} .
$$

Then $\left\{p_{\chi} ; \chi \in \hat{G}\right\}$ is a family of mutually orthogonal projections in $A_{G}$ with $\sum_{\chi \in \hat{G}} p_{\chi}=1$. Hence

$$
h\left(A_{G} \mid B\right) \geq \sum_{\chi \in \hat{G}}\left(\tau \eta E_{B}\left(p_{\chi}\right)\right)=\sum_{\chi \in \hat{G}} \eta\left(\frac{1}{|G|}\right)=|\hat{G}| \frac{1}{|G|} \log (|G|)=\log |G|,
$$

and we have that

$$
h\left(A_{G} \mid B\right)=\log |G| .
$$

In the next section, we show that inner conjugacy classes of subfactors $N$ of type $\mathrm{II}_{1}$ factor can take the maximum value of $h\left(N \mid u N u^{*}\right)$. 


\subsection{Case of $G=\mathbb{Z}_{n}$}

Here, we assume that the group $G$ in 3.1 is a finite cyclic group $\mathbb{Z}_{n}$, that is, $M$ is the crossed product $N \rtimes_{\alpha} \mathbb{Z}_{n}$ of a $\mathrm{II}_{1}$-factor $N$ by the group generated by an automorphism $\alpha$ on $N$ such that $\alpha^{n}$ is the identity and $\alpha^{i}$ is outer for $i=1, \cdots, n-1$. Such an automorphism $\alpha$ is called a minimal periodic automorphism (cf. [5]).

\subsubsection{Matrix units for minimal periodic automorphisms}

Let $\gamma$ be a primitive $n$-th root. Connes showed in the proof for the characterization of minimal periodic automorphisms ([5, Cor. 2.7]) that if $\alpha$ is minimal periodic, then there exists a set of matrix units $\left\{e_{i j}\right\}_{i, j=1}^{n}$ in $N$ such that

$$
\alpha\left(e_{i j}\right)=\gamma^{i-j} e_{i j}, \quad(i, j=1, \cdots, n) .
$$

Let $w=\sum_{i} e_{i+1, i}$. Then $w$ is a unitary in $N$ which satisfies that

$$
w^{j}=\sum_{i} e_{i+j, i}, \quad w^{i *} e_{j j} w^{i}=e_{j-i, j-i} \quad \text { and } \quad \alpha(w)=\gamma w .
$$

The following indicates that the inner congugacy class of $N$ can take the $\max _{L} h(N \mid L)$, where $L$ is a subfactor of $M$ with $[M: L]=n$.

Theorem 3.2.2. Let $N \subset M$ be the above. Then there exists a unitary operator $u$ in $M$ which satisfies the following properties :

(1) $h\left(N \mid u N u^{*}\right)=H_{N}(\operatorname{Ad} u)=\log n$.

(2) The conditional expectations $E_{N}$ and $E_{u N u^{*}}$ commute.

Proof. Let $w \in N$ be the unitary operator in 3.2.1. Let $v \in M$ be a unitary in $M$ implimenting $\alpha$, that is, $\alpha(x)=v x v^{*}$ for all $x \in N$. We put

$$
u=\frac{1}{\sqrt{n}} \sum_{i} w^{i} v^{i-1} .
$$

Then $u$ is a unitary and $\sqrt{n} E_{N}(u)=w$. Since $H_{N}(u)=\log n$, we have by Theorem 3.1.4 that

$$
h\left(N \mid u N u^{*}\right) \leq \log n
$$


As a finite partition of the unity, we choose $\left\{p_{j}: p_{j}=e_{j j}, j=1, \cdots, n\right\}$. Then

$$
\begin{aligned}
& h\left(N \mid u N u^{*}\right) \\
& \geq \sum_{j} \tau \eta E_{u N u^{*}}\left(p_{j}\right)-\tau \eta\left(p_{j}\right)=\sum_{j} \tau \eta E_{N}\left(u^{*} p_{j} u\right) \\
& \left.=\sum_{j} \tau \eta\left(\sum_{i} \alpha^{-i}\left(\frac{w^{i *} p_{j} w^{i}}{n}\right)\right)=\sum_{j} \tau \eta\left(\frac{\sum_{i} p_{j-i}}{n}\right)\right) \\
& =\log n
\end{aligned}
$$

Hence $h\left(N \mid u N u^{*}\right)=\log n$.

(2) To show that $E_{u N u^{*}} E_{N}=E_{N} E_{u N u^{*}}$, remark that for all $a \in N$,

$$
E_{u N u^{*}}\left(a v^{k}\right)=\frac{1}{n^{2}} \sum_{j}\left(\sum_{i, l} \gamma^{k^{2}+2 k i-(j+l) k-j l} w^{j+l-k-i} \alpha^{j+l-k-i}(a) w^{i-l}\right) v^{j} .
$$

Assume that $k \neq 0$. Then $E_{N}\left(a v^{k}\right)=0$. On the other hand,

$$
E_{N} E_{u N u^{*}}\left(a v^{k}\right)=\frac{1}{n^{2}} \gamma^{2 k} w^{-k} \alpha^{-1}\left(\sum_{i} \sum_{l} \gamma^{2 k i-l k} w^{l-i} \alpha^{l-i}(a) w^{i-l}\right)
$$

and

$$
\sum_{i} \sum_{l} \gamma^{2 k i-l k} w^{l-i} \alpha^{l-i}(a) w^{i-l}=\sum_{j}\left(\sum_{i} \gamma^{k(i-j)} w^{j} \alpha^{j}(a) w^{* j}\right)=0 .
$$

Therefore,

$$
E_{N} E_{u N u^{*}}\left(a v^{k}\right)=0=E_{u N u^{*}} E_{N}\left(a v^{k}\right) .
$$

for all $a \in N$ and $k=1, \cdots, n-1$. Also for each $a \in N$, we have that

$$
\begin{aligned}
& E_{u N u^{*}} E_{N}(a) \\
& \quad=\frac{1}{n^{2}} \sum_{j}\left(\sum_{i, l} \gamma^{j l} w^{j+l-i} \alpha^{j+l-i}(a) w^{i-l}\right) v^{j}=\frac{1}{n^{2}} \sum_{l} \sum_{i} w^{i} \alpha^{i}(a) w^{* i} \\
& \quad=\frac{1}{n} \sum_{i} w^{i} \alpha^{i}(a) w^{* i}=E_{N}\left(\frac{1}{n} \sum_{i} w^{i} \alpha^{i}(a) w^{* i}\right) \\
& =E_{N} E_{u N u^{*}}(a) .
\end{aligned}
$$


These show that

$$
E_{u N u^{*}} E_{N}(x)=E_{N} E_{u N u^{*}}(x) \text { for all } \quad x \in M
$$

\subsubsection{A continuous family of subfactors with index 2}

At the last, in the case of $G=\mathbb{Z}_{2}$, we show a result corresponding one in (4) for maximal abelian subalgebras of the type $\mathrm{I}_{n}$ factos $M_{n}(\mathbb{C})$.

Theorem 3.2.3. Let $N$ be a type $I I_{1}$ factor and let $M$ be the crossed product $N \rtimes_{\alpha} \mathbb{Z}_{2}$ by an outer automorphism $\alpha$ with the period 2. For the unitary $w \in N$ in 3.2.1, let

$$
u(\lambda)=\sqrt{\lambda} w+\sqrt{1-\lambda} v, \quad(0 \leq \lambda \leq 1) .
$$

Then $u(\lambda)$ is a unitary in $M$ which satisfies the followings :

$$
h\left(N \mid u(\lambda) N u(\lambda)^{*}\right)=H_{N}(u)=\eta(\lambda)+\eta(1-\lambda) .
$$

and

$$
\left\{h\left(N \mid u(\lambda) N u(\lambda)^{*}\right): \lambda \in[0,1]\right\}=[0, \log 2]
$$

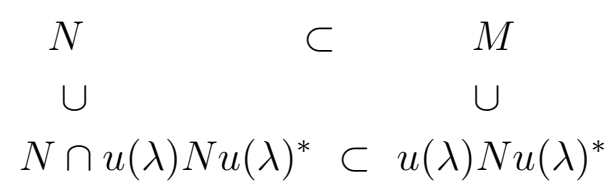

is a commuting square in the sense of [9] if and only if $\lambda=\frac{1}{2}$.

$$
h\left(N \mid u\left(\frac{1}{2}\right) N u\left(\frac{1}{2}\right)^{*}\right)=H_{N}\left(A d u\left(\frac{1}{2}\right)\right)=\max _{u} h\left(N \mid u N u^{*}\right)=\log 2
$$

where $u$ is a unitary in $M$. 
Proof. (1) It is clear that $H_{N}(A d u(\lambda))=\eta(\lambda)+\eta(1-\lambda)$. Hence by Theorem 3.1.4, we have

$$
h\left(N \mid u(\lambda) N u(\lambda)^{*}\right) \leq \eta(\lambda)+\eta(1-\lambda) .
$$

We remark that for each $x \in N$,

$$
E_{u(\lambda) N u(\lambda)^{*}}(x)=u(\lambda) E_{N}\left(u(\lambda)^{*} x u(\lambda)\right) u(\lambda)^{*}=\lambda w^{*} x w+(1-\lambda) \alpha(x) .
$$

Let $\left\{e_{i j}\right\}_{i, j=1,2} \subset N$ be the set of matrix units for $\alpha$ in 3.2.1. Then

$E_{u(\lambda) N u(\lambda)^{*}}\left(e_{i i}\right)=\lambda w^{*} e_{i i} w+(1-\lambda) \alpha\left(e_{i i}\right)=\lambda e_{i+1, i+1}+(1-\lambda) e_{i i}, \quad(\bmod 2)$.

Hence, we have that for each $i=1,2$,

$$
\tau \eta\left(E_{u(\lambda) N u(\lambda)^{*}}\left(e_{i i}\right)\right)=\frac{1}{2}(\eta(\lambda)+\eta(1-\lambda)),
$$

so that

$$
h\left(N \mid u(\lambda) N u(\lambda)^{*}\right) \geq \eta(\lambda)+\eta(1-\lambda) .
$$

This implies that

$$
h\left(N \mid u(\lambda) N u(\lambda)^{*}\right)=\eta(\lambda)+\eta(1-\lambda) .
$$

(2) First we remember the following; the diagram is a commuting square in the sense of [9] means that $E_{N} E_{u N u^{*}}=E_{u N u^{*}} E_{N}$.

Let $x \in N$. Since $\alpha(w)=-w^{*}$, we have that

$$
E_{N} E_{u(\lambda) N u(\lambda)^{*}}(x)=\lambda^{2} x+2 \lambda(1-\lambda) w \alpha(x) w^{*}+(1-\lambda)^{2} x
$$

and

$$
\begin{aligned}
& E_{u N u^{*}} E_{N}(x) \\
& \quad=\lambda^{2} x+2 \lambda(1-\lambda) w \alpha(x) w^{*}+(1-\lambda)^{2} x+\sqrt{\lambda(1-\lambda)}(2 \lambda-1) x w v
\end{aligned}
$$

Hence $E_{N} E_{u(\lambda) N u(\lambda)^{*}}(x)=E_{u N u^{*}} E_{N}(x)$ for all $x \in N$ if and only if $\lambda=1 / 2$. Similarly, for all $x \in N$,

$$
E_{N} E_{u(1 / 2) N u(1 / 2)^{*}}(x v)=w \alpha\left(x_{1}\right)+x_{1} w^{*}+x_{1} \alpha(w)+\alpha\left(w^{*} x_{1}\right)=0
$$

and

$$
E_{u(1 / 2) N u(1 / 2)^{*}} E_{N}(x v)=0 .
$$


These imply the conclusion.

(3) Since $N$ is a subfactor of $M$ with $[M: N]=2$, we have that

$$
h\left(N \mid u N u^{*}\right) \leq H\left(N \mid u N u^{*}\right) \leq H(M \mid N)=\log 2
$$

so that

$$
h\left(N \mid u\left(\frac{1}{2}\right) N u\left(\frac{1}{2}\right)^{*}\right)=\log 2=\max _{u} h\left(N \mid u N u^{*}\right) .
$$

\section{References}

[1] M. Choda, Entropy for *-endomorphisms and relative entropy for subalgebras, J. Operator Theory, 25 (1991), no.1, 125-140.

[2] M. Choda, Entropy for canonical shifts, Trans. Amer. Math. Soc., 334 (1992), no.2, 827-849.

[3] M. Choda and F. Hiai, Entropy for canonical shifts II, Publ. Res. Inst. Math. Sci., 27 (no.3) (1991),461-489.

[4] M. Choda, Relative entropy for maximal abelian subalgebras of matrices and the entropy of unistochastic matrices, Int. J. Math., 19(2008), no. 7, $767-776$.

[5] A. Connes, Periodic automorphisms of the hyperfinite factor of type $\mathrm{II}_{1}$, Acta Sci. Math., 39 (1977), 39-66.

[6] A. Connes, Entropie de Kolmogoroff Sinai et mechanique statistique quantique, C. R. Acad. Sci.Paris, 301 (1985), 1-6.

[7] A. Connes and E. Størmer, Entropy of $I_{1}$ von Neumann algebras, Acta Math., 134 (1975), 289-306.

[8] D. Evans and Y. Kawahigashi, Quantum Symmetries on Operator Algebras, Oxford Mathematical Monographs, Oxford(1998).

[9] F.M. Goodman, P. de la Harpe and V.F.R. Jones, Coxeter Graphs and Towers of Algebras, Math. Sciences Res. Inst. Publ., Springer Verlag (1989). 
[10] F. Hiai, Entropy for canonical shifts and strong amenability, Internat. J. Math., 6 (1995), 381-396.

[11] V.F.R. Jones, Index for subfactors, Invent. Math., 72 (1983), 1-25.

[12] V.F.R. Jones, Two subfactors and the algebraic decomposition of bimodules over $I I_{1}$ factors, Acta Math. Vietnam., 33 (2008), 209-218.

[13] H. Kosaki, Relative entropy of states : a variational expression, J. Operator Theory, 16 (1986), 335-348.

[14] S. Neshveyev and E. Størmer, Dynamical entropy in operator algebras, Springer-Verlag, Berlin(2006).

[15] R. Okayasu, Relative entropy for abelian subalgebras, To appear in Internat J. Math..

[16] M. Ohya and D. Petz, Quantum entropy and its use, Texts and Monographs in Physics. Springer-Verlag, Berlin(1993).

[17] D. Petz, A. Szántó and M. Weiner, Complementarity and the algebraic structure of 4-level quantum systems, J. Infin. Dim. Anal., Quantum Probability and Related Topics 12(2009), 99-116.

[18] M. Pimsner and S. Popa, Entropy and Index for subfactors, Ann. Sci. Ecole Norm. Sup., (4), 19 (1986), 57-106.

[19] S. Popa, Orthogonal pairs of *-subalgebras in finite von Neumann algebras, J. Operator Theory 9 (1983), $253-268$.

[20] E. Størmer, Entropy of endomorphisms and relative entropy in finite von Neumann algebras, J. Funct. Anal. 171 (2000), 34 - 52.

[21] K. Życzkowski, M. Kuś, W. Słomczyński and H.-J. Sommers, Random unistochastic matrices, J. Phys., A36 (2003), 3425-3450.

[22] Y. Watatani and J. Wierzbicki, Commuting squares and relative entropy for two subfactors, J. Funct. Anal., 133 (1995), 329-341.

The author was supported in part by JSPS Grant No.20540209. 